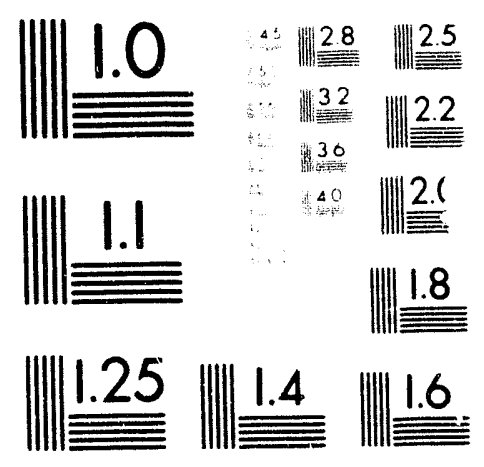



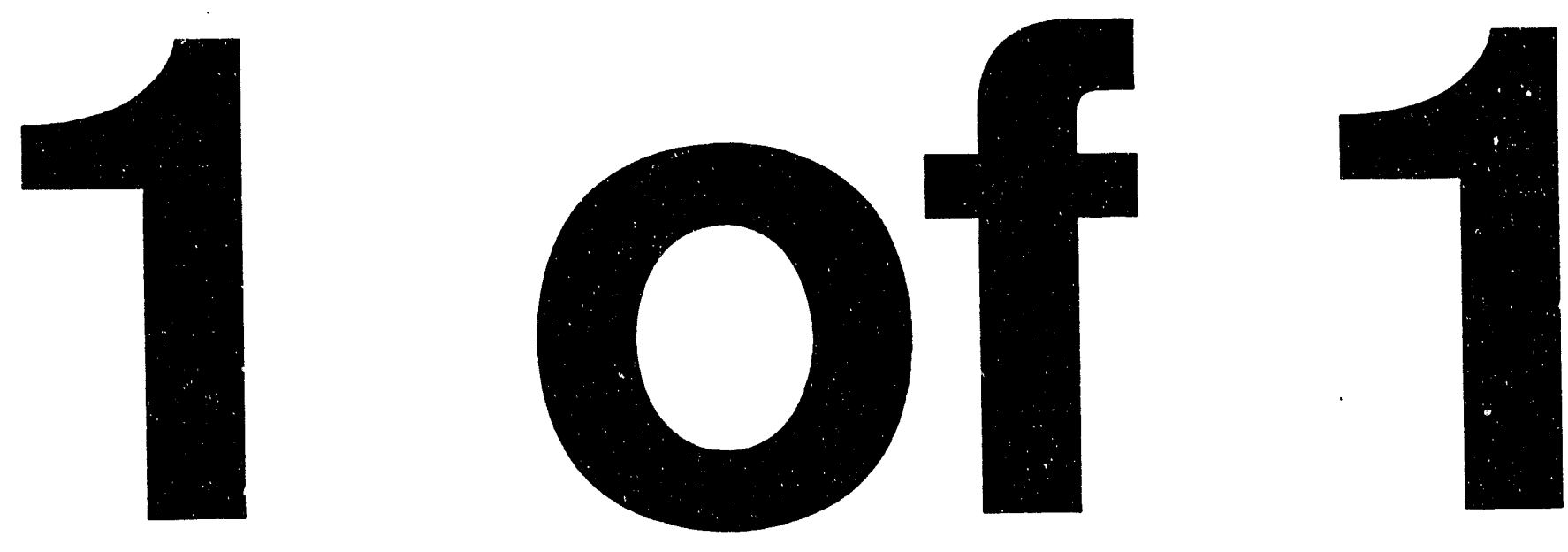

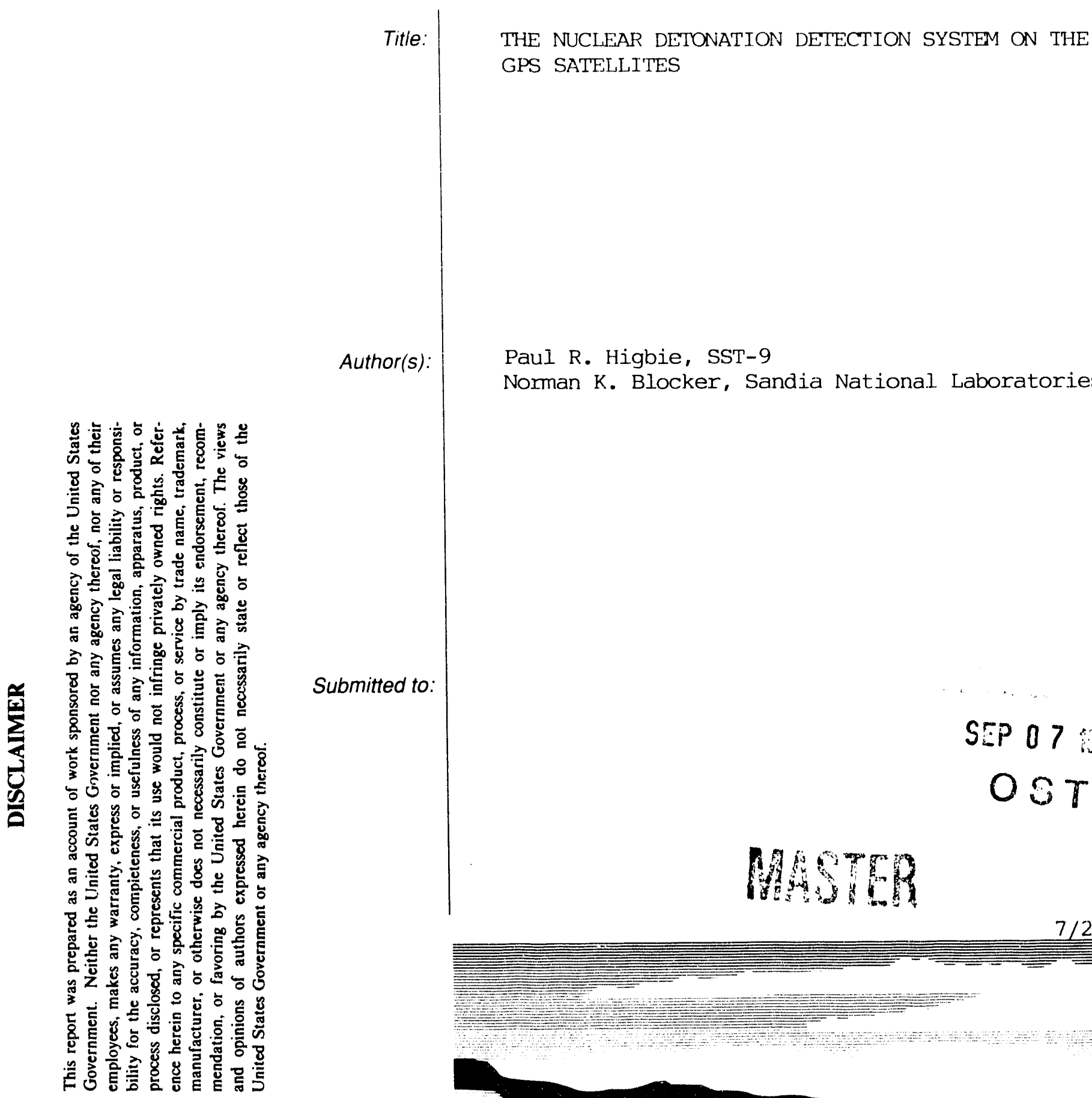

Paul R. Higbie, SST-9

Norman K. Blocker, Sandia National Laboratories

\section{SEP 07 \\ 0.071}

\section{Pag}

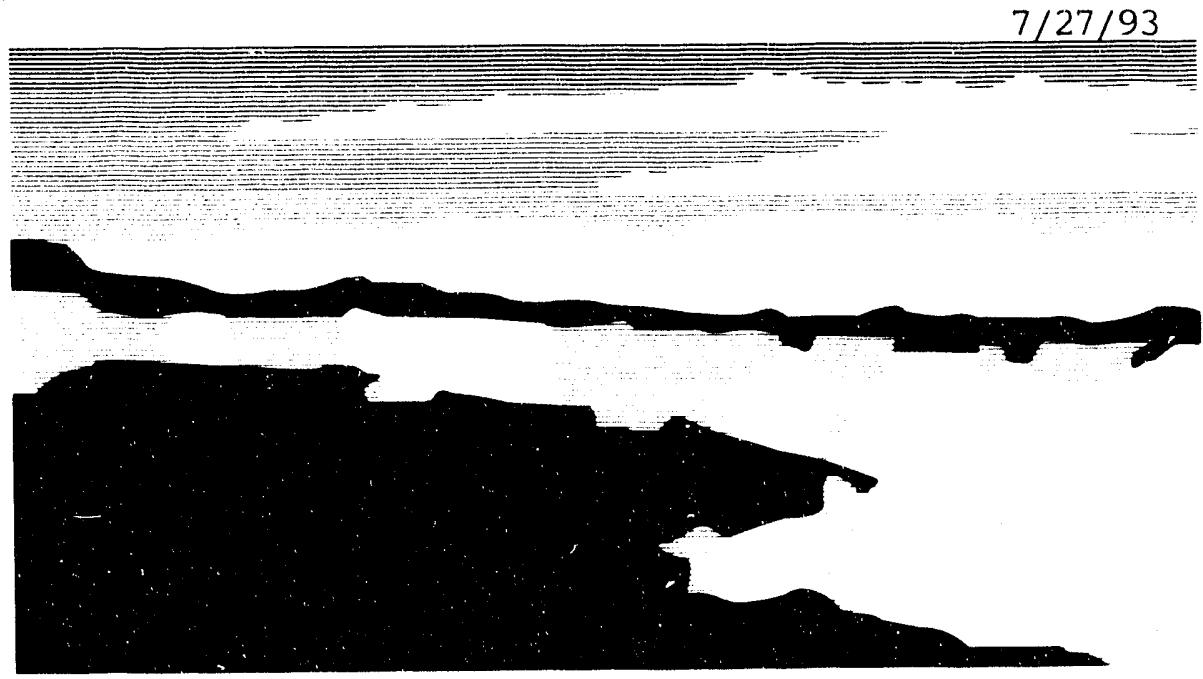

NATIONAL LABORATORY

Los Alamos National Laboratory, an affirmative action/equal opportunity employer, is operated by the University of California for the U.S. Department of Energy under contract W-7405-ENG-36. By acceptance of this article, the publisher recognizes that the U.S. Government retains a nonexclusive, royalty-free license to publish or reproduce the published form of this contribution, or to allow others to do so, for U.S. Government purposes. The Los Alamos National Laboratory renuests that the nuthlichar indentity this article as work pertormed under the auspices of the U.S. Department of Energy. 


\title{
THE NUCLEAR DETONATION DETECTION SYSTEM ON THE GPS SATELLITES
}

\author{
Paul R. Higbie, Los Alamos National Laboratory \\ Norman K. Blocker, Sandia National Laboratories
}

It will soon be 30 years since the first Vela satellite was launched on October 17, 1963. The Vela satellites vere developed in a joint effort by the Air Force and the Atomic Energy Commission - the predecessor organization to the Department of Energy. When President Kennedy and Chairman Khrushchev signed the Limited Test Ban Treaty (LTBT), one of the conditions was that each party to the treaty could monitor the ban on testing in the atmosphere or in space using its own technical means. Vela was based on the considerable experience obtained from developing the measurement instruments for the rockets flown during the Dominic series of high-altitude nuclear tests conducted between April 25 and November 1962. Vela could therefore be a quick response to the LTBT, which was signed on August 5, 1963. Figure 1 shows President Kennedy inspecting a mockup of the Vela satellite; he visited Los Alamos National Laboratory and Sandia National Laboratories, Albuquerque in December 1962.

The relationship between the United States and the members of the former Soviet Union has improved greatly since the early steps taken by Kennedy and Khrushchev. However, nuclear technology and modern techniques are widely diffused throughout the world. Should the NonProliferation Treaty, which bans nuclear weapons development by its signatories fail, the United States, as the world's remaining superpower, still has the capability to detect clandestine nuclear tests conducted anywhere.

Part of the United States' capabilities are carried on the GPS satellites. The fact that the GPS satellites have the capability of detecting nuclear detonations has never been classified, although it has not been well advertised either. In this article we will give a qualitative overview of the GPS capabilities. The GPS Nuclear Detonation Detection System is-as Vela was- a joint program between the Air Force and the Department of Energy. The Air Force provides the "platform," the GPS satellites in this case, and operates the system, while the Department of Energy, through its National Laboratories, Sandia and Los Alamos, provides the sensors.

The physical output-light, gamma rays, $x$-rays, neutrons-from a nuclear explosion, as well as the secondary effects due to the interactions with the atmosphere of these primary forms of energy output, are well known. The standard reference for a detailed discussion of these phenomena is still Glasstone's book The Effects of Nuclear Weapons, first published in 1950. (The third edition, 1977, is available from the U. S. Government Printing Office.) The measurements of the outputs of an event using instruments sensitive to different phenomena helps prevent misidentifying an event due to some natural phenomenon (e.g., a lightning flash) as a nuclear detonation.

The sensors on the GPS satellites have been designed to measure the outputs due to various phenomena. In particular, visible light, radio waves, and $x$-rays are measured by sensors on the satellites. In addition, background measurements of the radiation environment are also performed by instruments on some of the GPS satellites. The GPS orbit at 4.1 RE runs through the most intense part of the radiation belts.

Figure 2 is a photograph of the $\mathrm{x}$-ray instrument that was flown on the GPS Block I satellites. It is a simple sensor that is designed to measure the intense burst of $\mathrm{x}$-rays that would accompany the exoatmospheric detonation of a nuclear weapon. Such a burst would occur in an interval of less than 1 microsecond, so the sensors must continuously monitor 
for such an event. Should such an event occur, data from all the satellites observing the event are transmitted to ground terminals for processing.

Because the $x$-rays from a nuclear detonation would travel outward from the location of the event in what would be essentially a spherical shell expanding at the speed of light, the location of the event can be determined from accurate timing information as part of the measurement at each responding satellite. This case is essentially the reverse of the navigation problem, where a receiver at a fixed point receives accurate time marks from several satellites to determine its location. In the first case, the detonation acts as an impulse transmitter. The time differences of arrival (TDOA) at four or more satellites must be measured to calculate the time of the event and its $x-y-z$ coordinates.

In addition to providing the timing information needed to calculate the location, the sensor itself measures the intensity of the $x$-rays impinging upon it. This information, together with range obtained with the TDOA technique described above, can be used to calculate the yield of the device that was detonated.

For adequate coverage, most of the satellites in the constellation of 24 satellites have $x$-ray detectors on them. However, a few satellites in the constellation have a background sensor - to measure the natural particle fluxes in the radiation belts through which the satellites pass-substituted for the $\mathrm{x}$-ray instrument. This background information is important to assure the proper interpretation of data from the $x$-ray and other sensors. The background instrument's interface to the spacecraft is identical to that of the $x$-ray instrument so that the differences between the two are invisible to the spacecraft manufacturer. The essential difference is that the background monitor operates continuously, providing copious amounts of data-which is not desired with the $x$-ray sensor.

Of the constellation of 24 GPS satellites, 3 have a background instrument onboard instead of a $\mathrm{x}$-ray instrument. Figure 3 is a photograph of the head of a Block I background instrument showing holes in a composite dome designed to reduce the background fluxes of energetic electrons to acceptable counting-rate levels. The holes allow a sampling of the fluxes over one hemisphere. A filter at the bottom of the holes provides an energy threshold so that the dose can be measured for a certain range of equivalent radiation shielding. Besides providing information for interpretation of data from other sensors and also providing information on the total dose received by the satellites, the data from these sensors are valuable for scientific purposes. The background sensors measure a region of the magnetosphere not routinely investigated and the long span of data available makes a valuable scientific data set, albeit the instruments are very crude by the standards of most scientific instruments.

Sandia National Laboratories has developed an optical radiometer, called a bhangmeter, which records signals from nuclear explosions in the atmosphere. The most prominent effect of atmospheric nuclear explosions is the formation of a fireball whose light intensity, as it appears to a distant observer, undergoes fluctuations that result in two distinct peaks (cf. Glasstone, p.41). Detection of these peaks is a critical step in the identification of those optical events generated by nuclear explosions. The bhangmeter is a non-imaging radiometer that continuously monitors the full earth disk for these double-peaked signals. When a flash of light within its field of view exceeds a preset level and exhibits other characteristics of nuclear explosion signals, the bhangmeter triggers and records the optical intensity history. 
Bhangmeters, like other optical instruments on earth-orbiting satellites, respond to many signals that have norhing to do with nuclear explosions. The most significant of these is reflected sunlight from the earth. A fully sunlit earth disk illuminates the sensor with more than ten thousand times the intensity seen from some nuclear explosions. The slowly varying signal from the earth-reflected sunlight is electronically suppressed to permit the sensor to detect the much dimmer but fast-rising signal from a nuclear explosion. Transient false alarm signals result from lightning, an event that occurs many thousands of times each day over the earth, and from sun glints off water surfaces. False signals can also result when high-energy particles strike the bhangmeter's detector elements. The bhangmeter is designed to reject these false triggers, thereby maintaining a high probability of detection of atmospheric nuclear explosions.

Calibration of the GPS satellite bhangmeters is accomplished using a ground-basec ruby laser system located at Sandia. Fast-rising laser pulses are accurately time-tagged using GPS clocks at the laser site and their time-of-arrival is recorded by the bhangmeter system aboard the satellite. The result obtained from this procedure provides an end-to-end test of all the processing functions involved in the calculation of satellite positions and corrections to timing data. Data sets from different satellites are combined to evaluate the location capability of the optical sensors by the TDOA location technique described above. Figure 4 shows a green neodymium beam being transmitted from the Sandia laser site with the lights of Albuquerque as a backdrop.

Intense radio waves are also generated by a nuclear explosion. This phenomenon arises from energized electrons (Compton currents) produced by the interaction of the primary bomb radiation with the atmosphere. The Conipton currents are turned by the Earth's magnetic field to produce an electromagnetic pulse (EMP). The satellite carries a specialized antenna and set of electronics to detect and measure the EMP from a nuclear detonation. Figure 5 is a photograph of a ground-based antenna developed at Los Alamos that is used to stimulate the EMP detector on the GPS satellites.

The United States has the technological capability to determine whether an emerging nuclear power has conducted a test of a nuclear weapon. The same detection system can be used to monitor the progress of a nuclear war, should that ever occur. Until the time comes when the political shape of the world will have so changed that the possibility of the use of nuclear weapons will have disappeared, such systems as the GPS/NDS are vital to the nation's security. 


\section{FIGURES}

Fig. 1 President Kennedy viewing the Vela satellite. Other figures in the photograph include Glenn Seaborg, Chairman of the AEC; McGeorge Bundy, the National Security Advisor; and S.P. Schwartz, President of the Sandia National Laboratory.

Fig. 2 The $x$-ray instrument for the Block I GPS satellites. This is a simple detector consisting of a filter in the form of a dome that reduces the flux of particles passing through it and thereby reduces the background. Dual sensors behind the dome provide redundancy for the instrument.

Fig. 3 Four domes of the background instrument. The thick domes are used to reduce the counting rate to a limit that can be conveniently handled by the electronics. The holes allow a sampling of the flux over a hemisphere. A hemispherical filter inside each of the domes defines an energy threshold for the particles entering the holes. Thus four energy channels can be defined for the dose measured by the instrument.

Fig. 4 Test firing of a neodymium laser pulse at the Sandia calibration facility. Actual calibration pulses for the bhangmeter use a short pulse from a ruby laser.

Fig. 5 Antenna for the calibration of the EMP monitor on the GPS satellites. This facility is at Los Alamos National Laboratory. During calibration, the antenna is directed at the satellite, and then a short burst of RF energy is radiated towards the satellite. 


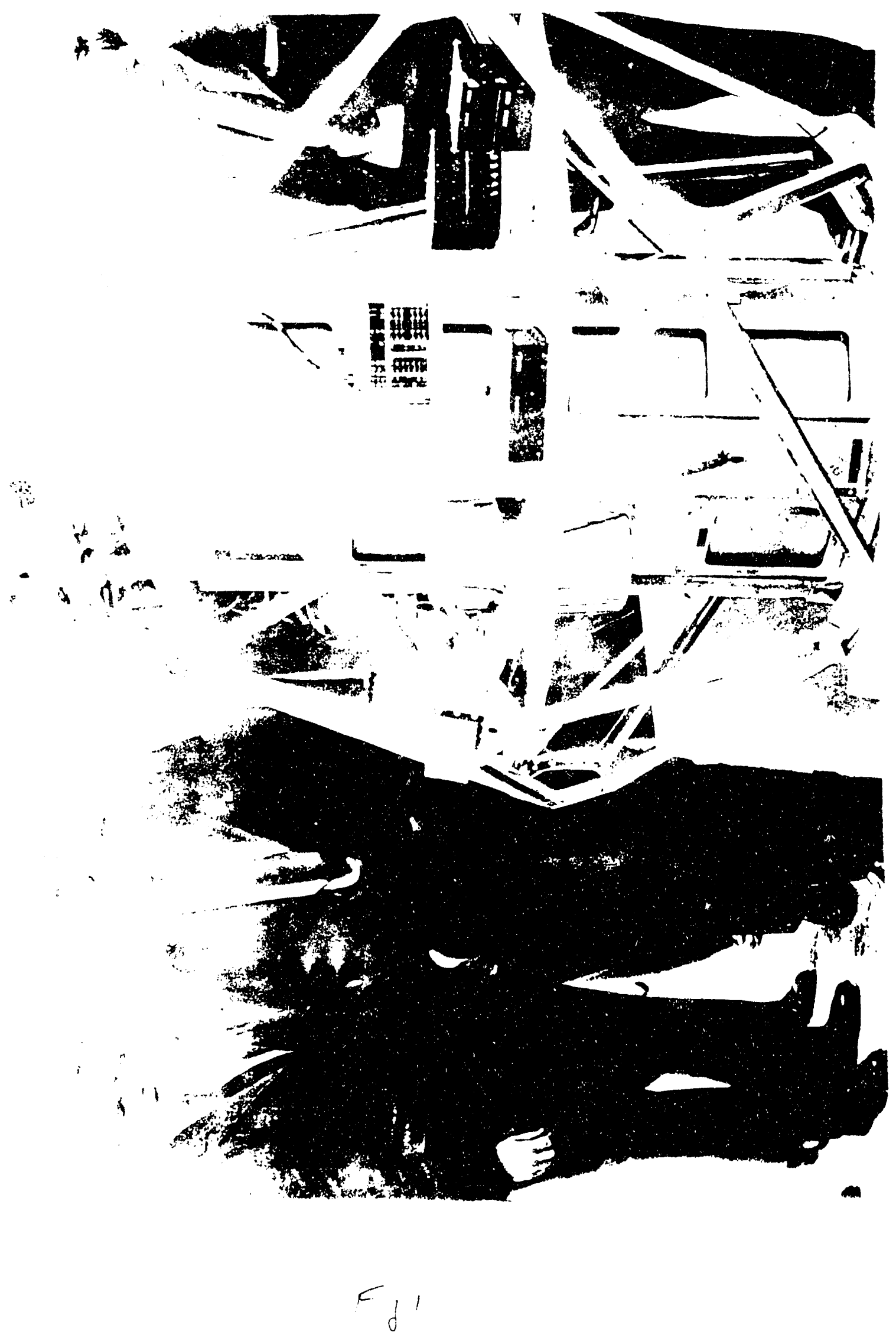




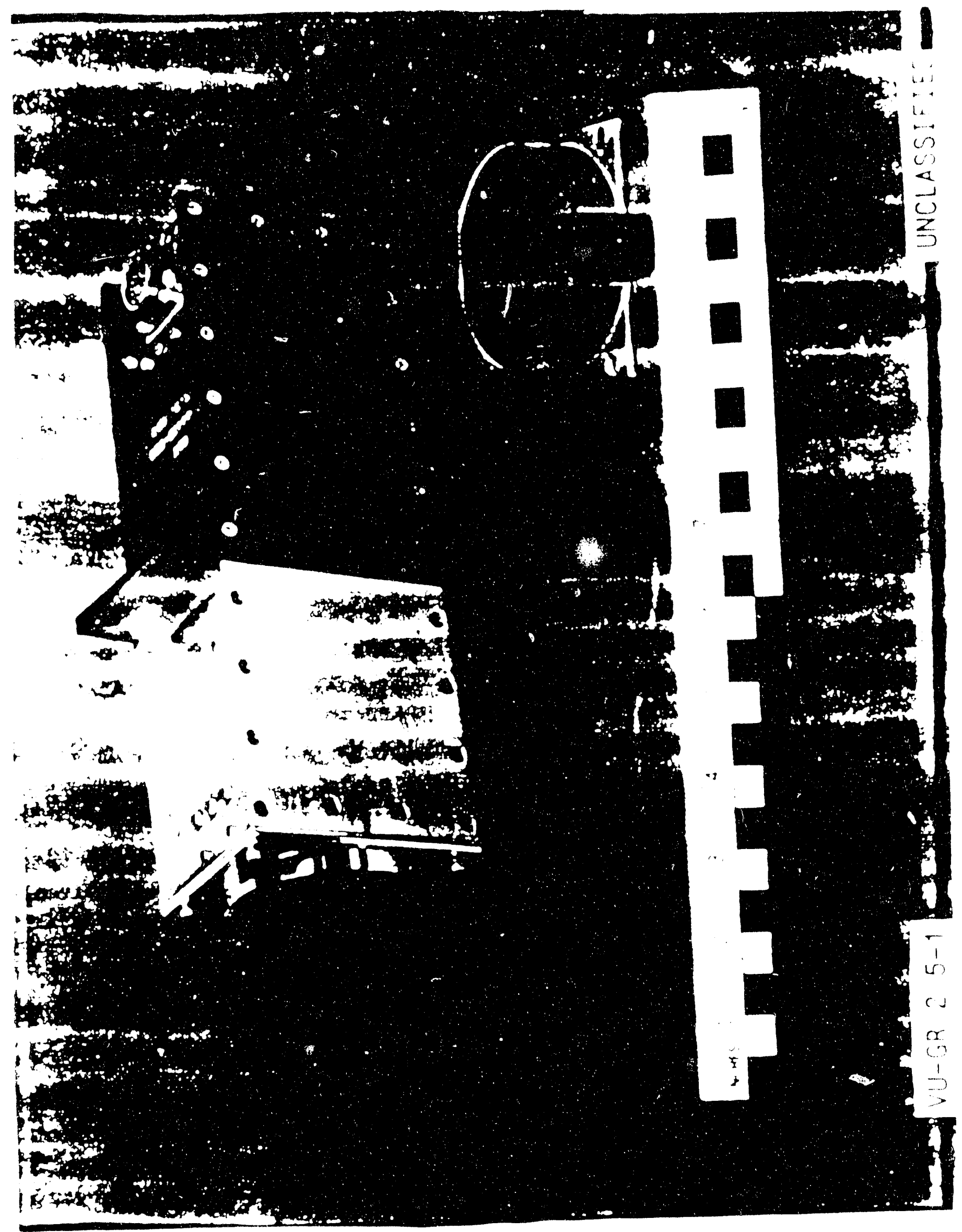




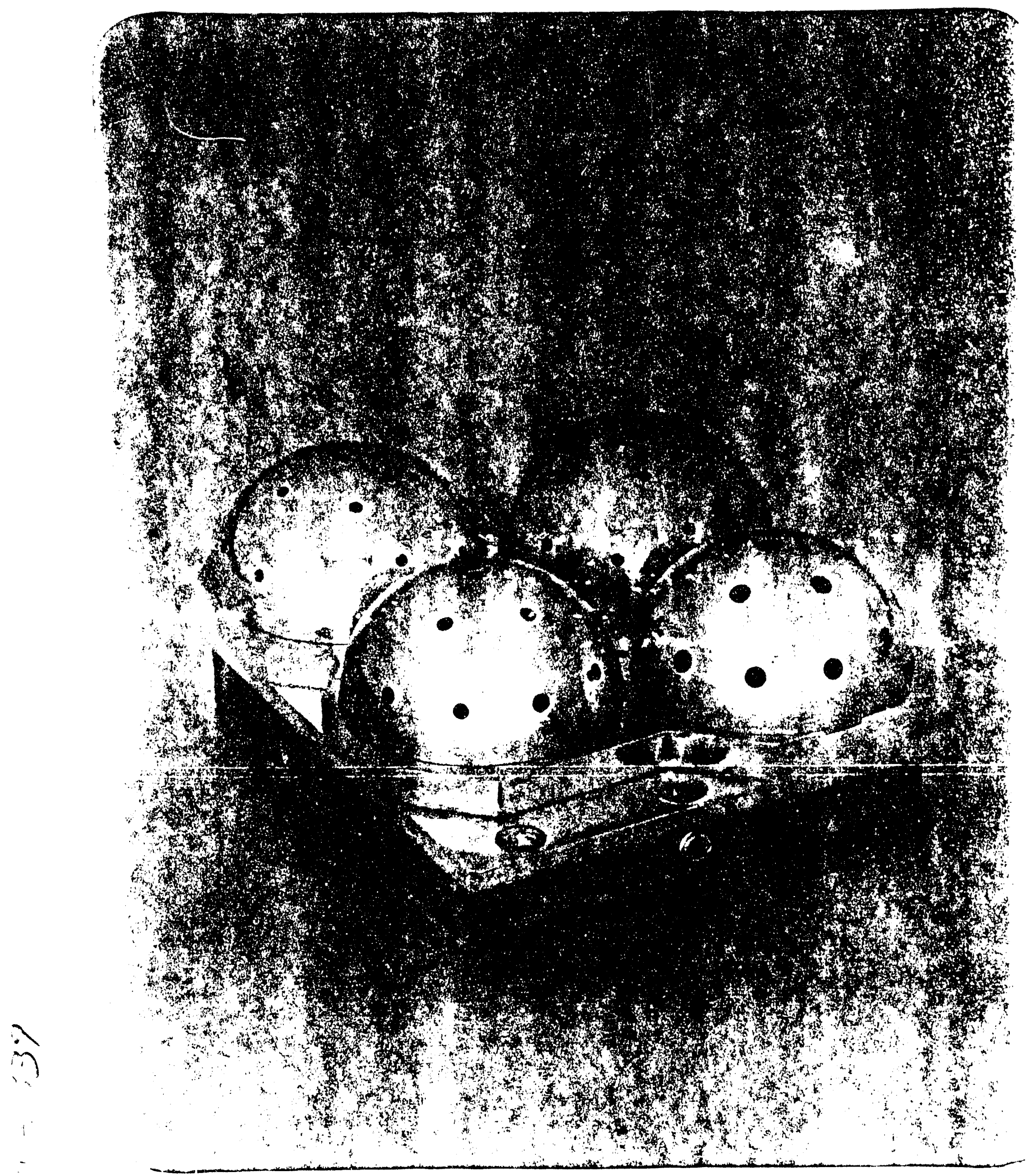




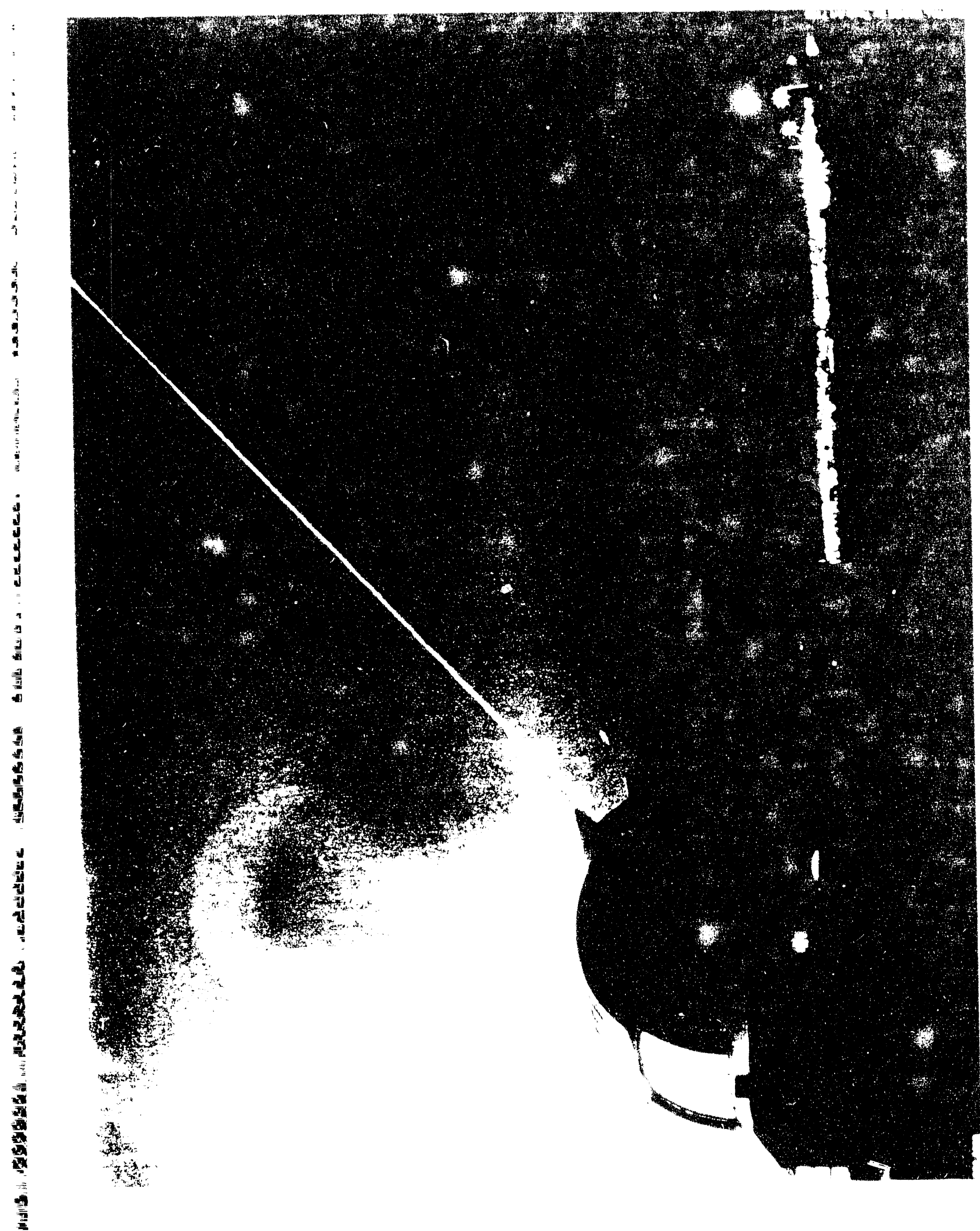




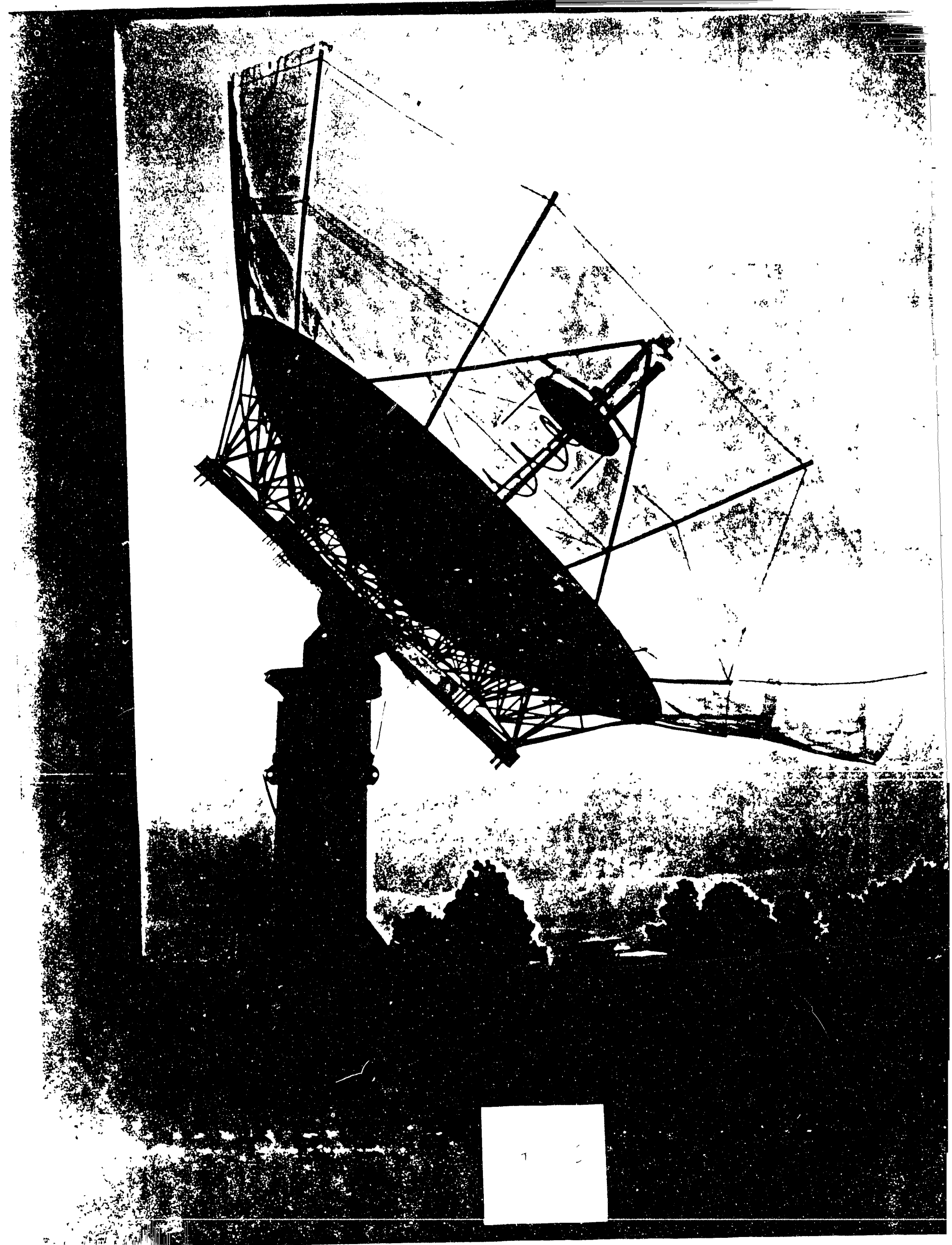



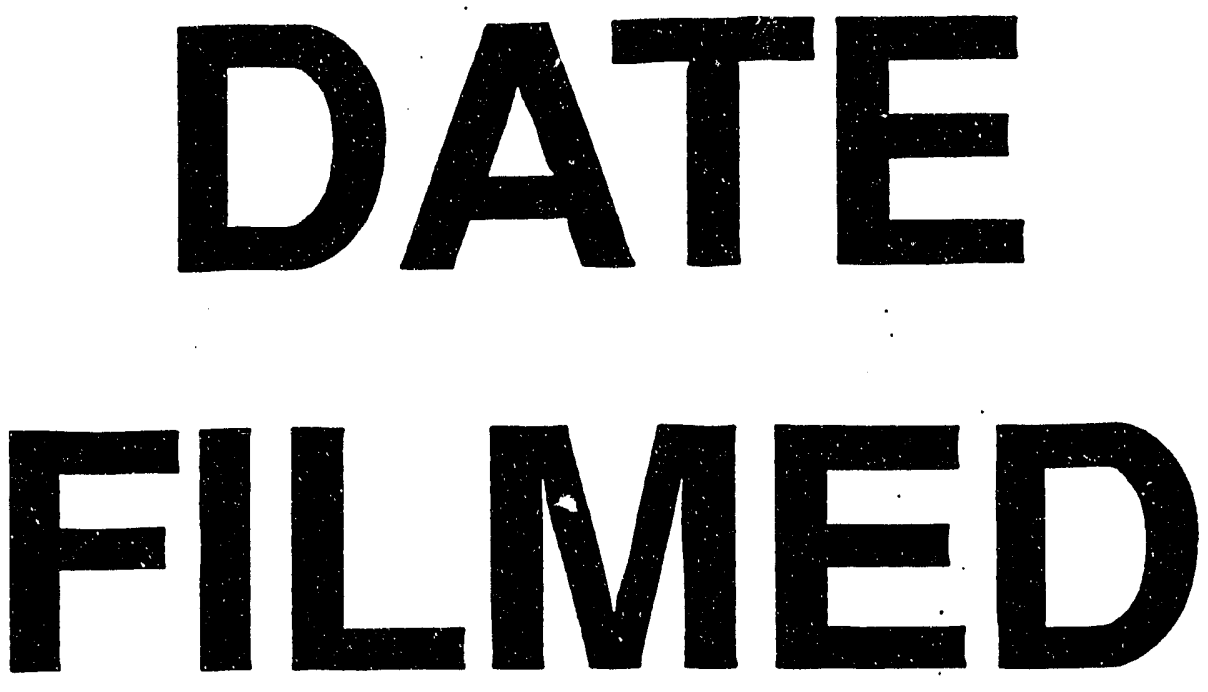

$11 / 8 / 93$
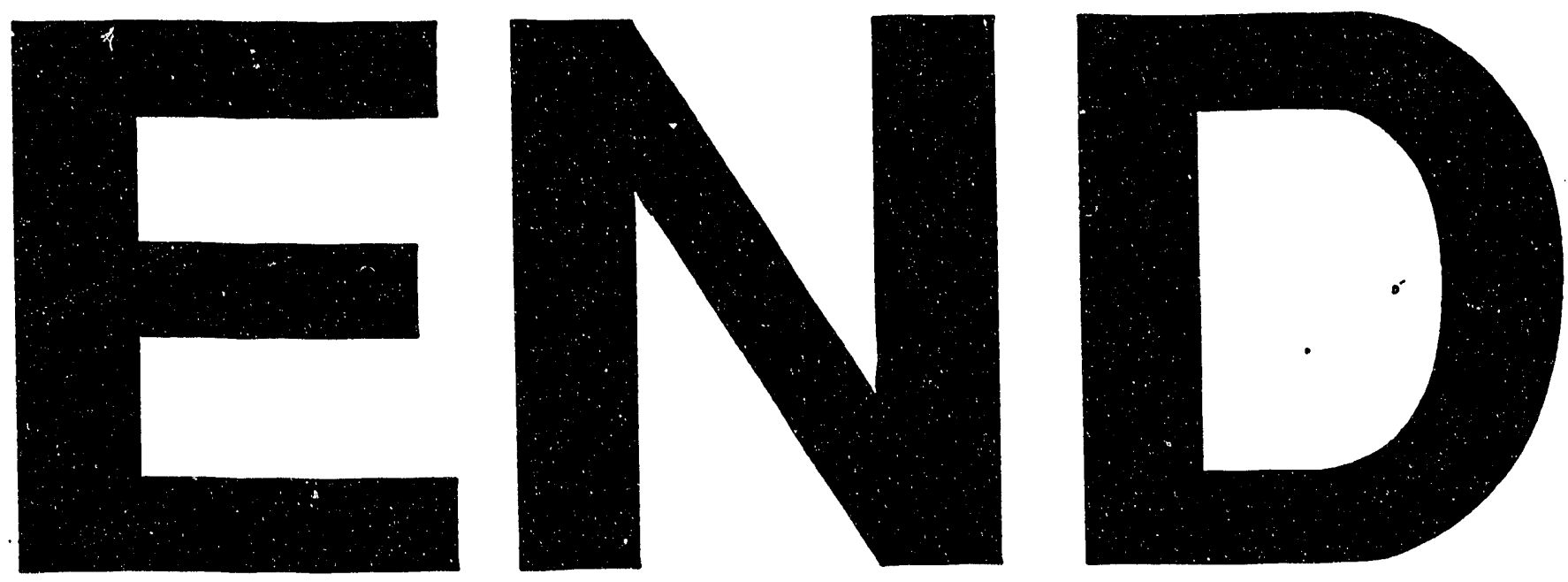